\title{
A MONOLITHIC FINITE ELEMENT APPROACH TO COMPUTE PERMEABILITYAT MICROSCOPIC SCALES IN LCM PROCESSES
}

\author{
L. Silva ${ }^{1,2^{*}}$, G. Puaux ${ }^{1}$, M. Vincent ${ }^{1}$, P. Laure ${ }^{1}$ \\ ${ }^{1}$ MINES ParisTech - CNRS UMR 7635 - CEMEF \\ ${ }^{2}$ Faculdade de Engenharia da Universidade do Porto - DEMEGI
}

\begin{abstract}
In this paper, a numerical approach for permeability determination at the mesoscopic and microscopic scales is proposed. In an eulerian framework, the computational domain (corresponding to the elementary cell) is composed of one single mesh, where the interface between the fibres (microscopic scale) or the yarns composing the fabric (mesoscopic scale) and fluid is captured through a level set approach. At the microscopic scale, Stokes equations are considered. At the mesoscopic scale, resolution of a coupled Stokes (in the fluid)-Darcy (in the yarn) flow is necessary and is performed using also a mixed finite element technique, providing a single system of equations. Stabilization of the Brinkman flow is attained using the $\mathrm{P} 1+/ \mathrm{P} 1$ element. Results on permeability computation at the microscopic scale, as well as sensitivity analysis, illustrate the methodology followed.
\end{abstract}

KEYWORDS: permeability computation, monolithic approach, mixed finite elements

\section{INTRODUCTION}

LCM (Liquid Composite Moulding) processes involve the injection of a resin matrix in a mould where a fibrous reinforcement has been placed. One of the main problems arising in the process is the formation of porosities as a consequence of the bad impregnation of the resin. Simulation is used at the process scale to optimise the process, but does not predict the distribution of the porosity, since current macroscale models do not include the multi-scale nature of the fibrous media (Figure 1). Fibre reinforcements are composed of several yarns that gather multiple fibres.

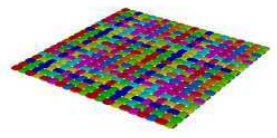

a) Part

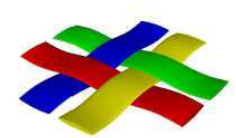

b) Yarns

Mesoscopic

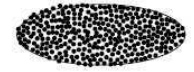

c) Fibres

Microscopic
Figure 1: Three-scale modelling of fibre reinforced media

At the macroscopic scale, one solves the Darcy equation by considering a homogeneous porous media. Nevertheless, permeability used at this scale may be more accurately determined by using direct simulation at the mesoscopic and microscopic scales on REV (Representative Elementary Volumes), object of this paper.
Hence, at the fibre scale yarn permeability can be determined using an immersed volume technique and by solving Stokes equations, by considering that fibres are not permeable. At the yarn scale, a similar approach is used, but Brinkman equations are solved in the whole computational domain.

Computations have been performed using CIMLib, the scientific computation library developed at CEMEF, on which REM3D, a polymer and composite injection moulding software is built from.

\section{IMMERSED VOLUME TECHNIQUE}

We consider the computational domain composed of both the reinforcement (fibres or yarns) and the resin. Thus, a multidomain problem is considered, with two phases, solid and fluid. A monolithic approach [1] has been chosen: computation is performed using a single mesh that includes all the phases; interfaces between them are known implicitly through a distance function $\alpha$ to these interfaces (Figure 2).

\subsection{MIXTURE LAW}

Material properties, such as density or viscosity, must be computed on the whole computational domain. A linear mixture law for the viscosity has been used:

$$
\begin{aligned}
& \eta=\eta_{s} H_{\alpha}(\alpha)+\eta_{f}\left[1-H_{\alpha}(\alpha)\right] \\
& \frac{\eta}{K}=\left(\frac{\eta}{K}\right)_{s} H_{\alpha}(\alpha)+\left(\frac{\eta}{K}\right)_{f}\left[1-H_{\alpha}(\alpha)\right]
\end{aligned}
$$

\footnotetext{
* Corresponding author: MINES ParisTech - CNRS UMR 7635 - CEMEF, Rue Claude Daunesse 06904 - Sophia-Antipolis France, Phone: +33 (0)4 93678944 - Fax: +33 (0)4 923897 52, e-mail: Luisa.Silva@mines-paristech.fr
} 
where $H_{\alpha}(\alpha)$ is the modified Heaviside function

$$
H_{\alpha}(\alpha)=\left\{\begin{array}{ccc}
1 & \text { if } & \alpha>e \\
\frac{\alpha}{e} & \text { if } & 0<|\alpha|<e \\
0 & \text { if } & \alpha<-e
\end{array}\right.
$$

In these equations, $\eta$ is the viscosity, $\alpha$ is the distance function to the fibre-fluid interface (positive inside the fibre or yarn) and $e$ is the half-thickness of the mixture zone. The indexes $s$ and $f$ indicate the solid (fibre or yarn) and fluid (resin) domains, respectively.
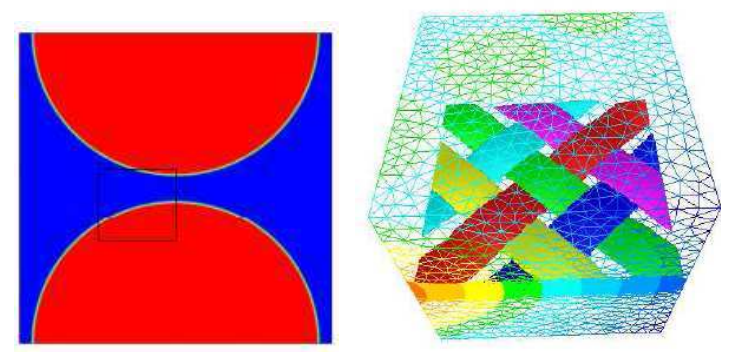

Figure 2: Example of computational domains (REVs) at the micro (left) and meso (right) scales. In the left, the micro-scale: red represents the fibre and blue the resin. In the right, interfaces between yarns and resin are represented through the isosurface of zero value of $\alpha$ on the background mesh

\subsection{MESH ADAPTATION}

To guarantee a good accuracy on the description of the fibre-resin and yarn-resin interfaces, mesh control is necessary. Thus, anisotropic mesh refinement at the interface level allows the control of the number of elements in the thickness $e$, as well as their orientation. To do that, an anisotropic metrics field is computed on the mesh, defining the mesh size in each spatial direction and is given to the mesher, incorporated in our solver.

Being our interface defined by the gradients of a distance function, the metrics field better adapted is

$$
M=m^{2}\left(\nabla \alpha \otimes \nabla \alpha^{T}\right)+\varepsilon^{2} I
$$

where $I$ is the identity tensor, and the mesh sizes in directions $\nabla \alpha$ and $\nabla \alpha^{T}$ are $1 / \sqrt{ }\left(m^{2}|\alpha|^{2}+\varepsilon^{2}\right)$ and $1 / \varepsilon$. If we adapt the mesh only in the thickness $e$, the metrics field can be redefined as

$$
M=\left\{\begin{array}{ccc}
\varepsilon^{2} I & \text { if } & |\alpha|>e \\
\left(\frac{N}{e}-\varepsilon\right)^{2} \frac{\left(\nabla \alpha \otimes \nabla \alpha^{T}\right)}{|\nabla \alpha|^{2}}+\varepsilon^{2} I & \text { if } & |\alpha|<e
\end{array}\right.
$$

where $N$ is the number of desired layers in the thickness $e$. In this thickness, we have $N$ elements of size $e / N$ in the direction $\nabla \alpha$ and the default mesh size $1 / \mathcal{E}$ in the direction $\nabla \alpha^{T}$. Figure 3 illustrates adaptation at the fibreresine interface.

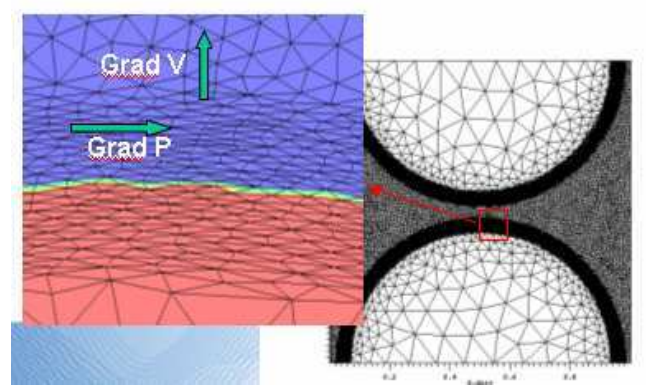

Figure 3: Example of anisotropic mesh adaptation at the fibre-resin interface.

\section{FLOW EQUATIONS}

\subsection{BRINKMAN EQUATIONS}

Darcy and Brinkman equations model flow through a porous media like flow through an equivalent continuous homogeneous media [2].

In the following, we suppose that the reinforcement is static and non deformable, the fluid is Newtonian, its density is constant and the media is saturated. Continuity equation, supposing that velocity at the pore surface is zero, is

$$
\nabla \cdot v=0
$$

where $\mathrm{V}$ is the average velocity field. Averaging conservation of momentum leads to the Brinkman equation

$$
-\frac{\phi \eta}{K} v+\eta \Delta v-\phi \nabla p=0
$$

with $p$ the pressure, $K$ the permeability and $\phi$ the porosity.

\subsection{THE MICROSCOPIC SCALE}

At the fibre-resin scale, we consider that $(\eta / K)=0$ in both the fiber and the resin, and we are led to the Stokes equations in the whole computational domain

$$
\left\{\begin{array}{l}
\eta \Delta v-\nabla p=0 \quad \text { in } \Omega \\
\nabla \cdot v=0
\end{array}\right.
$$

Rigidity of the solid (fibre) part is taken into account through a penalisation scheme, by imposing $\eta_{s}=10^{3} \eta_{f}$ [3].

\subsection{THE MESOSCOPIC SCALE}

At the yarn-resin scale, we consider that $(\eta / K)_{f}=0$ in the resin and that there is no viscous term $\eta \Delta v=0$ in the yarn. Thus, we are led to the Stokes equations in the fluid domain and to the Darcy one in the solid. Through our mixture law, one can solve one single problem, Brinkmans', in the whole computational domain

$$
\begin{aligned}
& -\frac{\phi \eta}{K} v+\eta \Delta v-\phi \nabla p=0 \text { in } \Omega \\
& \nabla \cdot v=0
\end{aligned}
$$




\subsection{NUMERICAL RESOLUTION}

Brinkmans' problem (8) is solved using a mixed finite element method. The element chosen is the $\mathrm{P} 1+/ \mathrm{P} 1$, which has a linear approximation in pressure and also a linear one in velocity, enriched with a pyramidal bubble function. This enrichment stabilises the formulation for both the Stokes and the Darcy cases. Indeed, the discrete weak formulation of (8) is, after part integration and using the bubble function properties: find $\left(v_{h}+b_{h}, p_{h}\right) \in\left(V_{h} \oplus B_{h}, P_{h}\right)$, such that

$$
\begin{aligned}
& \int_{\Omega_{h}} 2 \eta_{h} \varepsilon\left(v_{h}\right): \varepsilon\left(w_{h}\right)-\int_{\Omega_{h}} p_{h} \nabla \cdot w_{h}+\int_{\Omega_{h}}\left(\frac{\phi \eta}{K}\right)_{h}\left(v_{h}+b_{h}\right) w_{h}=0 \\
& \int_{\Omega_{h}} 2 \eta_{h} \varepsilon\left(b_{h}\right): \varepsilon\left(b^{\prime}{ }_{h}\right)-\int_{\Omega_{h}} p_{h} \nabla \cdot b^{\prime}{ }_{h}+\int_{\Omega_{h}}\left(\frac{\phi \eta}{K}\right)_{h}\left(v_{h}+b_{h}\right) b^{\prime}{ }_{h}=0 \\
& -\int_{\Omega_{h}} q_{h} \nabla \cdot v_{h}-\int_{\Omega_{h}} q_{h} \nabla \cdot b_{h}=0
\end{aligned}
$$

$\forall\left(w_{h}+b_{h}{ }_{h}, p_{h}\right) \in\left(V_{h} \oplus B_{h}, P_{h}\right) . \quad V_{h}, B_{h}, P_{h} \quad$ are the discrete functional spaces associated to the linear velocity and pressure, and to the bubble function. For more details on these spaces, see [1]. The element contribution to the linear system arising can be written in the matrix form:

$$
\left[\begin{array}{ccc}
A_{v v}{ }^{T} & A_{v b} & A_{v p} \\
A_{v b}{ }^{T} & A_{b b} & A_{b p} \\
A_{v p}{ }^{T} & A_{b p}{ }^{T} & 0
\end{array}\right] \cdot\left[\begin{array}{c}
v_{h} \\
b_{h} \\
p_{h}
\end{array}\right]=\left[\begin{array}{l}
0 \\
0 \\
0
\end{array}\right]
$$

Bubble condensation provides a system in the main variables $v_{h}$ and $p_{h}$ :

$$
\left[\begin{array}{cc}
A_{v v}-A_{v b} A_{b b}{ }^{-1} A_{v b}{ }^{T} & A_{v p}-A_{1 b} A_{b b}{ }^{-1} A_{b p} \\
A_{v p}{ }^{T}-A_{b p}{ }^{T} A_{b b}{ }^{-1} A_{v b}{ }^{T} & -A_{b p}{ }^{T} A_{b b}{ }^{-1} A_{b p}
\end{array}\right] \cdot\left[\begin{array}{c}
v_{h} \\
p_{h}
\end{array}\right]=\left[\begin{array}{l}
0 \\
0
\end{array}\right]
$$

where

$$
\left[\begin{array}{cc}
-A_{v b} A_{b b}{ }^{-1} A_{v b}{ }^{T} & -A_{1 b} A_{b b}{ }^{-1} A_{b p} \\
-A_{b p}{ }^{T} A_{b b}{ }^{-1} A_{v b}{ }^{T} & -A_{b p}{ }^{T} A_{b b}{ }^{-1} A_{b p}
\end{array}\right]
$$

is the stabilisation matrix. The linear system resulting from the discrete formulation is solved using a conjugate residual method and an ILU preconditioning, using the PETSC library, to obtain the nodal distributions of the velocity and pressure fields.

\section{PERMEABILITY COMPUTATION}

Once velocity and pressure have been computed, permeability can be determined (at the micro or meso scales). Three different techniques are most often used: the capillary model, models arising from the hydrodynamic lubrication theory and the cell model. Hydrodynamic lubrication theory models suppose the fibrous media as a regular cylinder spacing geometry and compute an analytical solution for the Stokes equations inside this REV (Representative Elementary Volume). The most popular one for the transverse permeability of the array of cylinders is Gebart's expression [4]:

$$
K_{\perp}=C_{1}\left(\sqrt{\frac{v_{f}^{\max }}{v_{f}}}-1\right)^{5 / 2} R^{2}
$$

where $R$ is the fibre radius, $v_{f}$ is the fibre volume fraction, $v_{f}^{\max }$ is the maximum fibre volume fraction and C1 a constant value, both defined for a square array of fibres.

In the cell model, one may consider any fibre array geometry (a cell), and Stokes equations are solved on the considered cell. Idris [5] gives a review on the numerical results obtained for the transverse permeability on triangular and square arrays of fibres, for Newtonian and pseudoplastic matrix fluids.

In our case, Darcy's equation, defining permeability, will be directly considered. Thus,

$$
K=\eta\left(\frac{\langle v\rangle}{\langle\nabla p\rangle}\right)_{f}
$$

where $\langle v\rangle$ and $\langle\nabla p\rangle$ are the averaged velocity and pressure gradient fields (only considered in the fluid domain). Thus, using equation (2)

$$
K=\eta \phi \frac{\int_{\Omega}(1-H(\alpha)) v}{\int_{\Omega}(1-H(\alpha)) \nabla p}
$$

This expression allows permeability computation on whatever REV, and its results are compared with Gebart's relation in the following, at the microscopic scale.

\section{RESULTS AND DISCUSSION}

We consider a regular array of fibres (Figure 4). A pressure gradient is imposed from left to right and the normal velocity in the upper and lower planes is zero. Inside the fibres, velocity is also zero. A sticky contact condition between fibres and the fluid is implicitly taken into account through the formulation. Three different sizes of REV where considered and the non-dimensional values of the transverse permeability obtained were: $\mathrm{K}(4$ fibres $)=0.0088, \mathrm{~K}(9$ fibres $)=0.0086, \mathrm{~K}(4$ fibres $)=0.0086$. Thus, one may consider that the 4-fibre geometry is the $\mathrm{REV}$, for a square geometry.
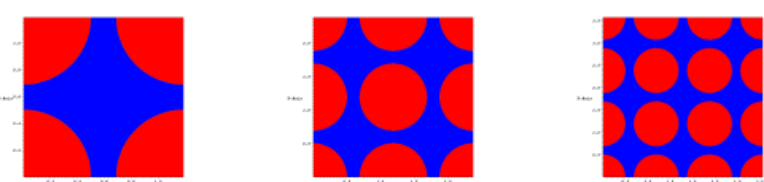

Figure 4: Different sizes of RVE for permeability computation

Sensitivity to the mesh size has been studied. Actually, two different meshes have been generated: one isotropic and another anisotropic adapted at the interface (Figure 5). For an identical accuracy on the permeability, 
anisotropic meshes have 10 times less elements than isotropic meshes (Figure 6).
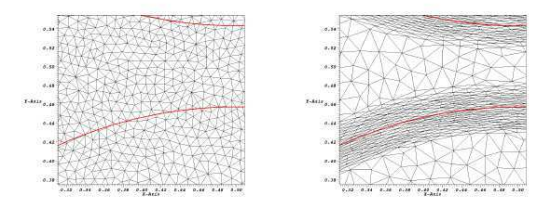

Figure 5: Mesh sensitivity analysis - Adapted meshes, on the left isotropic and on the right anisotropic (in red, the fibre-fluid interface)

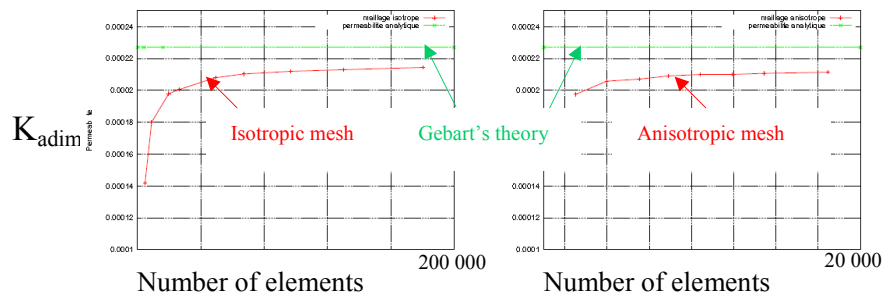

Figure 6: Mesh sensitivity analysis - Evolution of the permeability computed value (in red), for a total number of elements that vary from 5000 to 200000 in the isotropic case (left) and from 5000 to 20000 in the anisotropic one (right); in green, Gebart's value for the transverse permeability

Different types of arrays have been considered (Figure 7). Differences of $17 \%$ between the two transverse permeabilities have been found for geometry a, 5\% for geometry $\mathrm{c}$ and $1.7 \%$ for geometry $\mathrm{b}$ (reason for choosing this REV on the different tests performed). In fact, for multidomain computation, orientation of the REV and size is important.

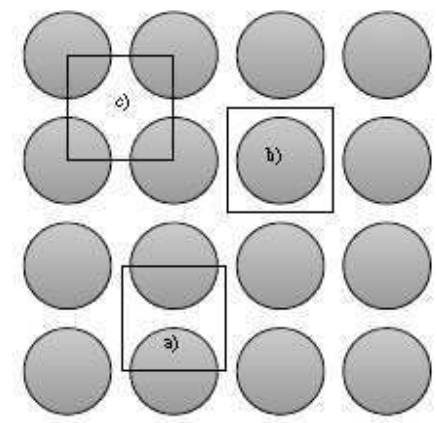

Figure 7: REV configurations studied.

Finally, to validate permeability computation, we study different fibre rates and compare our results with references from the literature [5]. Figure 8 shows that results are in good agreement (our method "monolithique").

\section{CONCLUSIONS}

Simulation of LCM processes at the macroscopic scale must include a relevant permeability tensor. To determine this tensor, permeability at the microscopic and then at the mesoscopic scale must be computed.

In this paper, a monolithic technique has been detailed to provide computation at both scales for whatever geometries of REV. Computations at the microscopic scale have been undertaken, showing a good agreement with the theoretical framework of permeability determination. Sensitivity analysis concerning mesh type and array size and type has been shown.

Current work includes the same testing at the mesoscopic scale using a similar approach, as well as the non saturated case (three-phase flow).

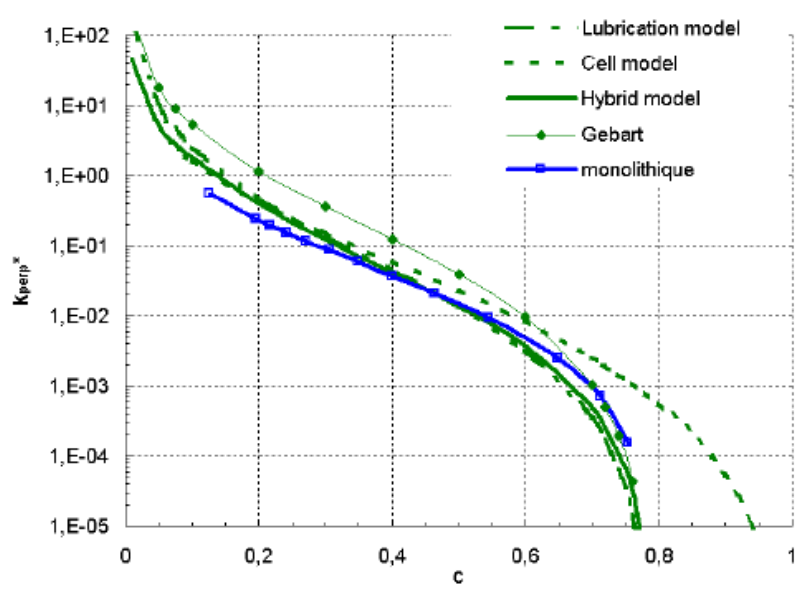

Figure 8: Evolution of the transverse permeability with the fibre volume fraction, our results are referenced as "monolithique".

\section{ACKNOWLEDGEMENT}

The authors acknowledge the ANR (Agence Nationale pour la Recherche, France) for the funding of this work, part of the project LCM3M.

\section{REFERENCES}

[1] T. Coupez, H. Digonnet, P. Laure, L. Silva, and R. Valette. Calculs éléments finis multidomaines: applications aux problèmes multiphasiques. In $\mathrm{M}$. Souli, editor, Interaction fluide-structure: modélisation et simulation numérique, pages 165201. Lavoisier, France, 2009.

[2] S. Whitaker : The Method of Volume Averaging. Kluwer Academic Publishers, 1999.

[3] P. Laure, G. Beaume, O. Basset, L. Silva, and T. Coupez. Numerical methods for solid particles in particulate flow simulations. European J. Comp. Mechs., 16:365-383, 2007.

[4] B.R. Gebart. Permeability of unidirectional reinforcements for RTM. J. of Comp. Mat., 26(8):1100-1133, 1992.

[5] Z. Idris. Modelling the flow of power-law fluids through anisotropic fibrous media. PhD thesis - INP Grenoble, France, 2006. 